\title{
ABOUT THE SPECTRAL CLASSIFICATION OF Be STARS
}

\author{
A. Feinstein \\ Observatorio Astronómico, La P1ata, Argentina. \\ H. Tignanelli \\ Observatorio Astronómico, La Plata, Argentina.
}

The problem of describing the De stars is especially interesting to investigate. A large collection of these objects are organized into information with preserving structures, such as classifications. In this case the difficulties consist of: 1) determining which are the main properties in the classification, and 2) deciding which one of the possible classifications should be used. For the first part there are a MK spectral system and a scheme of classifications presented by Jaschek et al. (1980), among others. The second part has been worked out in many ways. Here we describe, following the Information Theory (IT), an analytical method to compare both classifications of Be stars.

We apply the method proposed by Estabrook (1967) to the sample of 140 Be stars subdivided in five groups by Jaschek et al. (1980). It is possible to assign, to each of these classifications, a measure of entropy according to the IT. This is essentially a measure of how difficult it is to predict which one of the possible descriptions of the classifications is applicable to some object randomly choosen from a set of Be stars. Depending on their associated distributions of probability, different classifications will be assigned to different measures of entropy.

From our analysis:

1) We obtain 0.690 units of information shared in by both classifications, which means that both have in common a small portion of the information about the characteristics of Be stars.

2) The unconditional entropy of Jaschek's classification yields $H(J)=1.442$ units of information, and the corresponding to the MK classification $\mathrm{H}(\mathrm{T})=3.768$ units of information. That means that the exclusive information of Jaschek's classification is greater than the exclusive information of $\mathbb{M}$ spectral classification.

3) It is possible to compute a measure of the interdependence from confusing measures (entropies) with the following:

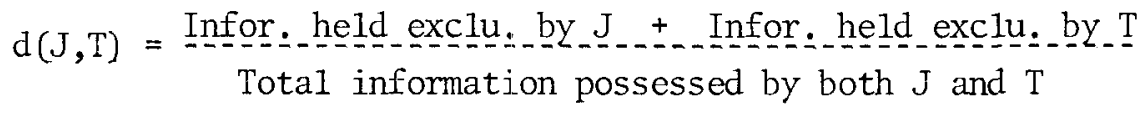

where $J$ denotes the Jaschek's classification and $T$ the $M K$ spectral classification. If $\mathrm{d}(\mathrm{J}, \mathrm{T})$ results equal zero, $\mathrm{J}$ and $\mathrm{T}$ are identical. On the contrary, if its value is one, $J$ and $\mathrm{T}$ are completely independent. 
Therefore, as the value obtained by us is $d(J, T)=0.883$, both classifications are highly independent. For that reason, to derive one group or state of one of the classifications, knowing the group or state in the other classification, has no meaning in terms of IT.

In conclusion, according to this comparison, we suggest that the characteristics of Be stars must be analyzed following the scheme given by Jaschek et al., because this one gives the minimal confusion (naximal information) and permits a complete description of the characteristics of the Be stars. Moreover, the simultaneous use of both classifications should be the result of undesirable redundancy in deriving properties of these objects.

REFERENCES:

Estabrook, G.F. (1967), Taxon, 16, 86.

Jaschek, M. , Hubert-Deplace, A. $\bar{M}_{\text {. }}$, Hubert, H. , Jaschek, C. (1980), Astron. Astrophys. Supp1. Ser., 42, 103.

\section{DISCUSSION FOLLOWING FEINSTEIN}

Abt :

You have shown that the Jaschek classification system is more useful in intercomparing Be stars. But if one wishes to compare $\mathrm{Be}$ stars with normal stars, shouldn't one use the MK system?

Feinstein:

The Jaschek system is useful only for Be stars, not for normal stars. 